\title{
Artigos \\ Aportes para una nueva experiencia de la seguridad en los estadios del fútbol argentino
}

\section{Aportes para uma nova experiência de segurança nos estádios de futebol argentinos}

Revista Brasileira de Tecnologias Sociais
1 Sociólogo (UBA) y Doctor en Antropología Social (UFSC). Desarrolló su tesis sobre las prácticas dirigenciales en el fútbol argentino. Fue docente de la Facultad de Ciencias Sociales de la UBA y es investigador y profesor en la Universidad Tres de Febrero (UNTREF). Es investigador del Núcleo de Antropología Visual y del Grupo de Antropología Urbana y Marítima ((NAVIGAUM-UFSC).

2 Licenciado en Sociología (UBA) y Magister en Ciencias Sociales (UBA). Es profesor e investigador de la Universidad Nacional de Tres de Febrero (UNTREF), de la Universidad Nacional de Avellaneda (UNDAV) y de la Universidad de Buenos Aires (UBA).

\section{Support for a new experience of security in Argentinian football stadiums}

\author{
Matías Godio $^{1} \&$ Santiago Uliana $^{2}$
}

Resumen: Este artículo es el resultado de una investigación orientada a formular nuevos paradigmas de acción e intervención en los fenómenos de violencia que se producen en los estadios del fútbol argentino. Por un lado, se retoman conclusiones de la extensa trayectoria de investigación de los autores en el campo de la violencia en el fútbol y sus dispositivos de seguridad. Por otro, se reflexiona desde el trabajo de campo de investigación-acción en la Provincia de Santa Fe, Argentina, en torno a las posibilidades de recrear prácticas culturales de convivencia y tolerancia entre los actores sociales involucrados: fuerzas de seguridad, espectadores/simpatizantes y diversas organizaciones de "hinchas".

Palabras clave: fútbol, seguridad, violencia, políticas públicas, espectáculo.

Resumo: Este artigo é o resultado de uma estratégia para desenvolver novos paradigmas de ação e intervenção nos fenômenos de violência que ocorrem nos estádios de futebol da Argentina. Se, por um lado, as conclusões da longa história de pesquisa dos autores no campo da violência no futebol e segurança são retomadas, por outro, elas refletem, a partir do trabalho de campo "pesquisa-ação", na província de Santa Fé, Argentina, sobre as possibilidades de recriar práticas culturais de convivência e tolerância entre os atores sociais envolvidos: forças de segurança, espectadores/torcedores e as torcidas organizadas.

Palavras-chave: Futebol, Segurança, Violência, Política Pública, Espetáculo.

Abstract: This article is the result of a strategy to develop new paradigms of action and intervention in the phenomena of violence that occur in Argentinian football stadiums. On one hand, this work also analyses the conclusions of the long history of research by authors in the field of violence in football, and security. On the other, it reflects, based on field work of "research-action" in the province of Santa Fé, Argentina, on the possibilities of recreating cultural practices to promote harmonious tolerance among the social actors involved: the security forces, the spectators/fans and the various 
organizations of "hinchas" i.e. football supporters.

Keywords: Football, Security, Violence, Public Policy, Public event.

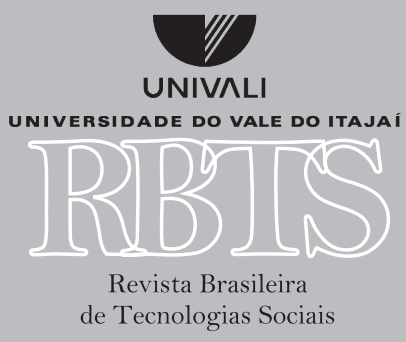


Los hechos de violencia asociados a los espectáculos de fútbol profesional en los estadios argentinos se han vuelto un problema recurrente y sistémico durante las última décadas. Este problema no ha logrado ser resuelto con eficacia y mucho menos originalidad por parte de las políticas públicas de seguridad. La virulencia, recurrencia y, hasta cierto punto, la estructuración de una lógica "violenta" integrada por barras bravas ${ }^{1}$ (hinchadas), simpatizantes (hinchas) y fuerzas de seguridad (policías) han determinado que en la última década se suspenda la presencia del público visitante en los estadios. Sin embargo, la violencia y las muertes han seguido rodeando el evento futbolístico, asumiendo diferentes formas y modalidades que inclusive, como lo muestran estudios recientes, han venido desplazándose de adentro hacia afuera del espectáculo propiamente dicho (ULIANA, SUSTAS, MURZI, 2011 ). Es decir, en otra temporalidad y otra espacialidad que no es la del partido y el estadio de fútbol. La necesidad de comprender el fenómeno de las formas que asumen las distintas violencias en el fútbol debe buscar nuevos caminos, pero sobre todo, integrar conocimientos académicos con experiencias prácticas de intervención sobre las dinámicas simbólicas y materiales que la estructuran.

Hemos aprendido de la antropología y la sociología contemporánea que los estadios de fútbol son espacios fundamentalmente polifónicos, y que dicha polifonía consiste en una variedad de discursos y prácticas que involucran una diversidad de actores sociales (BROMBERGER, 1998, 2001, 2004; GARRIGA, 2005, 2015; GIL, 2008). Estos actores son diversos y buscan obtener en el ritual futbolístico la experiencia de su dimensión polifónica, pública, plural y participativa. Es de esta forma que el ritual del evento futbolístico se asocia a la producción cultural de su vida cotidiana y de sus trayectorias sociales y culturales, es decir, se vincula con la subjetivación colectiva de variadas formas de experiencia del "estar en el mundo". En cierta forma, se trata de una teatralización de ese mundo proyectado en el evento espectacular.

En consecuencia, al rechazar e invalidar la violencia como hecho social y cultural dentro del estadio, no se consigue más que proyectarla hacia afuera, en una exterioridad e imposibilidad de teatralización de la misma. Son los procesos de conflicto territorial, las identidades locales, las apropiaciones de clase o status, las formas de pertenecer, la dinámica del poder y las diferencias de grupos sociales, de género y de rangos etarios -el fútbol es cada vez más una experiencia juvenil- aquellos que construyen esa polifonía a la que está abocado el fútbol en tanto ritual de representación en los estadios (GIL, 2002; RIAL, 2009; GODIO, 2010). Sobre él se lanzan projectos culturales y representaciones colectivas que entran en diálogo con formas particulares de gestionar y negociar procesos, pácticas y valores universales por los diversos actores, instituciones y grupos que los integran. ${ }^{2}$

En la Argentina el paradigma estatal de la seguridad se ha construido bajo sentidos culturales fuertemente estigmatizantes de los llamados "hinchas", favoreciendo el silenciamiento de la polifonía a la que antes aludimos. La explicación más común de los hechos violentos en los estadios de fútbol es alimentado por el periodismo de los grandes medios, y tiende fundamentalmente a reducir el papel de estos actores sociales al de una variación de sujetos "violentos" que operan como pares/espejos y que se enfrentan entre sí (ALABARCES, 2014). Esta visión del fenómeno se

1 Las 'barras bravas'constituyen una categoria émica en el fútbol argentino. Son formadas por grupos de simpatizantes que asisten en conjunto a los partidos de su equipo, tanto de local como de visitante. Generalmente ocupan un lugar privilegiado en las tribunas "populares" y definen su identificación con el club a partir da su capacidad de cantar y mostrar emblemas. A diferencia de Brasil, con las llamadas "torcidas organizadas", en la Argentina las barras no están legalizadas. Su organización interna y su estabilidad dependen de su capacidad de mantener enfrentamientos - y alianzas - con otras "barras".

2 Siguiendo a Guttman (2004) podemos decir que el fútbol sería una de las técnicas mediadoras en la construcción de epistemes locales, una sinopsis de la "racionalización romántica" de la categoría de récord en la competición deportiva. 
hace muchas veces explícita en los operativos de seguridad, los cuales están basados en "diagramas de control" policial orientados a la separación y que han venido a acentuar las rivalidades futbolísticas. En esa lógica se tiende a construir al "hincha" desde el sentido común como un sujeto cuyo significante se colma con categorías excluyentes y peligrosas. En esa percepción del otro, se dice que hay que "matarlo", "cogerlo" o que "no existe", metafóricamente en algunos casos y literalmente en otros. En este marco de absurda intolerancia respecto del otro -decimos absurda porque, como hemos remarcado, el fútbol necesita siempre de dos equipos que se enfrenten- la presencia de las fuerzas de seguridad es percibida por los distintos actores/espectadores con la misma animosidad (GALVAGNI, 2014). El paroxismo llega al punto de trasformar a las propias fuerzas de seguridad en un actor más dentro de ese juego de enfrentamientos excluyentes y violencia.

Es por ello que casi todo el dispositivo de seguridad se orienta a reducir los actores presentes a una condición de subalternos de las llamadas barras bravas, es decir, a enaltecer las formas más cohesionadas y autónomas de los llamados "hinchas" y transformar esa "totalidad" en el objeto privilegiado de represión y control de la violencia. Esto no solo tiende a reducir la polifonía y diversidad de los hinchas (que incluyen a las barras como una entre otras formas), sino también a convertir a las fuerzas de seguridad en una alteridad a la que referir sus actos, sus teatralizaciones, volviendo a pensar en los términos de Bromberger (2002). En consecuencia, la "garantía de seguridad" en los estadios de fútbol en la actualidad fomenta un dispositivo cultural que tiene como expresión primordial la militarización del espacio del estadio, centrando sus operativos de seguridad en la mortificación permanente de los espectadores como un tipo ideal de sujeto "violento" e "irracional" dentro y fuera del estadio (GODIO \& ULIANA, 2015). Esto se traduce sistemáticamente en una percepción negativa de los hinchas respecto de la policía y cualquier acción de los operativos de seguridad.

En los estadios de fútbol existe un ambiente de violencia que legitima las prácticas violentas de los espectadores. Estos últimos son sumergidos en la condición genérica de "delincuentes", "bárbaros" e "inadaptados" con la que se califica a las llamadas "barras bravas". Y en efecto, esta reducción colabora y mucho a que las acciones materiales y simbólicas de las que participan de distinto modo - más activa o pasivamente- estos hinchas, en general encuentren en las barras un motor privilegiado respecto de la producción de lenguajes y sentidos, donde la violencia forma parte constitutiva e impone condiciones a la proyección, por parte del Estado, de los operativos de seguridad.

\section{¿UN MODELO HEGEMÓNICO DE SEGURIDAD EN EL FÚTBOL?}

Una de las reformas más conocidas en los modelos de seguridad en los estadios de fútbol se gestó en Gran Bretaña durante las últimas tres décadas, hasta el punto de ser conocido hoy como el "modelo inglés". Esta reforma en los operativos de seguridad tuvo lugar tomando como punto de partida el Informe Taylor ${ }^{4}$, resultado de una amplia investigación encargada a las universidades inglesas sobre las causas y condiciones socioculturales que hacían posible la violencia en el fútbol de aquel país. Aun cuando es inadecuando reducir los fundamentos teóricos de esta perspectiva de

$3 \mathrm{La}$ actual cultura futbolera produce un relato que exalta la masculinidad por la vía de la violencia y que encuentra en las barras a uno de los principales precursores (no el único). Esta perspectiva de análisis tiene a los trabajos de Eduardo Archetti (2014) como referencia obligatoria.

4 El documento fue publicado en 1990 y lleva el nombre de su supervisor, Lord Taylor of Gosforth. Este enfoque toma forma luego del drama de Hillsborough en 1989, donde 96 hinchas del Liverpool mueren aplastados por una avalancha de gente. 
acción a una única línea de interpretaciíon académica de los fenómenos de violencia en el deporte, la extensa trayectoria de la sociología del deporte inglesa tuvo una fuerte influencia.

Gran parte de las medidas tomadas en Inglaterra para dar con la solución al problema se basaron entonces en la formulación de políticas públicas apoyadas en investigación de las ciencias sociales, cuyos resultados, dicho en forma genérica, sostuvieron que la violencia en el fútbol se produce como consecuencia de prácticas, relaciones y hábitos culturales que pueden modificarse e introyectarse en los sujetos a partir de la resignificación de los contextos en que se producen los eventos futbolísticos. En este sentido, la visión interaccionista de los procesos de subculturación asociados a las transformaciones de la estuctura social británica encontraron puntos de encuentro con la teoría configuracional de la Escuela de Leicester. Esta última argumentaba su perspectiva en la existencia de vínculos de determinación entre prácticas deportivas de competición y los procesos de construcción/resistencia de las subjetividades occidentales. Fue Norbert Elias quien instaló el foco del análisis en los sentidos y significaciones sociales de una economía política de las emociones, gestionada a través de los deportes de competición en las sociedades industriales. Esta línea de reflexión asumía la idea de la presencia de una micropolítica de las prácticas de los sujetos presente en los espectáculos deportivos asociada a la creación de “mensajes" de orden y racionalidad del lazo social. Es decir, el fútbol expresaría la búsqueda de la monopolización de los cuerpos y la violencia por parte del Estado (ELIAS, 1987; ELIAS \& DUNNING, 1992). Para Elias, las transformaciones de los juegos deportivos a partir de mediados del siglo XIX se relacionan con los cambios culturales de larga duración de las sociedades en el proceso civilizatorio moderno, siendo la violencia y la exaltación de los sujetos elementos a domesticar. ${ }^{5}$ Los fenómenos de violencia, por lo tanto, son comprendidos en una trama de sentidos y mensajes culturales que explicitan ese trayecto en un contexto social, económico y político más amplio que el de los estadios de fútbol.

La reinterpretación pragmática de esta perspectiva analítica permitió pensar nuevos objetos de intervención como las llamadas "subculturas" -los jóvenes, por ejemplo-, las cuales fueron situadas en el contexto del proceso civilizatorio asociado con los espectáculos de masas y con el deporte. Así, las ciencias sociales aplicadas en el Informe Taylor entendieron que las "soluciones" y "éxitos" del mencionado modelo debían centrarse esencialmente en la construcción de dispositivos culturales orientados a producir un pasaje del estadio como campo de batalla de identificaciones sociales, políticas y religiosas a un modelo de fútbol espectáculo y de consumo donde la dimensión represiva ocupaba también un rol importante, aunque no debía ser el único. Lo más importante del dispositivo no es la presencia policial ni las vallas o alambres que cierran los pasos y "conducen” hacia un lugar "seguro" la violencia potencial, sino en sumergir la imagen del hincha como expresión de identidades colectivas polifónicas en una totalidad fragmentada y parecerse, en un campo distinto, a la de individuos consumidores de shopping center o espectadores de cine (MIGNON, 1998). El consumo se transforma en una forma novedosa de control social, de ahí el interés en el mencionado pasaje.

Con el objetivo de disminuir la violencia en los estadios ingleses, las políticas de seguridad pública se focalizaron en la aplicación de dos baterías de acciones diferenciadas: por un lado, establecer un control capilarizado permanente de los hinchas con el uso de nuevas tecnologías (cámaras de video, sistemas de acceso, colocación de butacas en el estadio, policía especializada); y

5 Elias ha hecho mucho para comprender la relación entre violencia, espectáculo y fútbol. Ha demostrado que el fútbol en tanto juego propone a quienes lo practican un conjunto de reglas para resolver la disputa en un campo de juego. Por el contrario, para los hinchas en las tribunas esas reglas no están del todo claras, son difusas y varían con el tiempo, son una construcción de quienes asisten como espectadores. 
por el otro, recrear un mercado fuerte y vigoroso en torno al espectáculo, centralizado en dotar a los sujetos de una nueva subjetividad de consumidor, en este caso de fútbol. ${ }^{6}$

Como resultado de esto, en Inglaterra el fútbol se generalizó como una experiencia de consumo, siendo los estadios lugares - no lugares diría Augé (2000)- dominados por el modelo de experimentación y compra de bienes culturales bajo la proyección ideal de las clases medias y medias altas. Aquellos sectores de las clases populares que no se adaptan a ese modelo o no pueden pagar los nuevos costos del espectáculo dejaron de concurrir al estadio o se transformaron en "espectadores de sofá”.

Este modelo ha influenciado y dominado la perspectiva de seguridad en los espectáculos futbolísticos en toda Europa occidental y en gran parte de las ligas del mundo ${ }^{8}$. Modelo que tiene a su vez las Copas Mundiales de Fútbol y la Premier League en Inglaterra como eventos ejemplares de espectacularización e instancia de amplificación mediática global. Sirviéndonos de Debord (2005) es factible afirmar que esta modalidad de gestión de la seguridad es un aspecto “necesario” para la lógica/estética del espectáculo televisado.

Más cerca de nuestro país aparece Uruguay, quien ha venido incorporando elementos de este modelo hegemónico de seguridad. En ese país vecino se han introducido un conjunto de cambios en la seguridad deportiva relativamente novedosos para nuestra región. Se ha retirando la policía de dentro del estadio, hecho que ha buscado la transferencia de la responsabilidad a los clubes y a la Asociación Uruguaya de Fútbol, en tanto organizadores de un espectáculo al cual asiste el público, pero que es de "carácter privado". Este cambio va acompañado de dos tipos de medidas; por un lado, las legales administrativas (ajustes en el marco normativo) y por el otro, la aplicación de nuevos dispositivos tecnológicos, como las cámaras de reconocimiento facial. Este cambio -eliminación de la policía dentro de los estadios- trajo un marcado aumento en la preocupación de parte de los clubes y la Asociación respecto de la seguridad de los espectadores, y sobre todo el tener que hacerse responsables del espectáculo en relación al comportamiento de los hinchas del club. Los operativos construyen un sujeto asimilado como "menor de edad", incapaz inclusive de portar un cinturón o un encendedor dentro del estadio, los cuales muchas veces son retenidos en los cacheos.

\section{UN FALLIDO “MODELO ARGENTINO” DE SEGURIDAD EN LOS ESTADIOS}

La seguridad deportiva, tal como ha venido construyéndose desde la década de 1980, ha generado una constante división entre los espectadores dentro y fuera de los estadios. Esta perspectiva se ha afirmado en la suposición de que las situaciones de violencia entre hinchas son

6 Entre las medidas podemos mencionar la supresión de los alambrados que rodean al campo de juego, la obligatoriedad de que todo el público asistente se encuentre sentado, una mejora en los accesos a los estadios que permita la evacuación rápida en caso de ser necesario, el reemplazo de los agentes pertenecientes a la policía por los llamados "stewards" (civiles capacitados para organizar grandes grupos y mediar en caso de ser necesario, sin el perfil represivo que caracteriza a los agentes policiales, la prohibición de vender tickets en los estadios en el día de partido, y la priorización de la venta de abonos para la temporada completa, la aplicación del derecho de admisión en los estadios y la confección de un "registro de aficionados" que recoge información del público que asiste al fútbol, la implicación de los clubes en la vida social y comunitaria de la ciudad a la que pertenecen, poniendo en práctica programas de acción comunitaria (más detalles en MURZI y SEGURA TREJO, 2015).

7 Kumar (2004) se refiere con ese término a aquellos espectadores que han sido expulsados de los estadios y reagrupados como tales como observadores de fútbol televisado.

8 En Bélgica existió una instancia de diagnóstico del problema en los estadios establecida por instituciones o autoridades independientes, como fue el caso el Informe Taylor. En Francia, el Ministerio de Deportes también encargó informes sobre el estado del problema del hooliganismo en el país a expertos en el tema provenientes de distintas universidades. 
resultado directo de "rivalidades futbolísticas"9 atravesadas por conductas delincuenciales. Es cierto que existen conductas delictivas en las que están involucrados integrantes de las barras bravas, por ejemplo personas que cobran dinero y controlan los alrededores de los estadios para cuidar los autos de aquellos que asisten a los espectáculos futbolísticos (llamados “trapitos"), la reventa de entradas y la distribución de drogas. Sin embargo, este modo de mirar -y operar- el problema ha tenido un impacto negativo en la resolución de la violencia en los estadios, potenciándola y reproduciéndola. Como bien explicitan Murzi y Segura Trejo (2015), en la actualidad, hablar de políticas de seguridad deportiva en Argentina equivale a pensar en una gran cantidad de agentes policiales trabajando en sus horas extras, mal pagados, escasamente capacitados para actuar en este tipo de eventos, pero con poder para a reprimir hinchas. Como contrapartida, las hinchadas se vuelven cada vez más provocativas y desafiantes en cuanto a su capacidad de imponer un orden propio dentro y fuera de los estadios.

Aun así, el problema es más complejo. El modelo de seguridad deportivo no solo se reduce a la militarización de los estadios y al control pormenorizado de los sujetos tenidos como estereotipos de los "violentos" (barras bravas). El operativo responde a un dispositivo cultural en el que la seguridad es pensada y ejercida con premisas simbólicas y materiales que plantean el espectáculo como un todo destinado a la producción de un "clima de violencia" que naturaliza y reproduce los hechos que son definidos como "violentos".

Por un lado, los operativos y dispositivos adquieren un eje espacial, cuadriculando los espacios circundantes al territorio, creando embudos de seguridad, vallados, alambrados, llegándose en los últimos años a prohibir la presencia de espectadores simpatizantes de los clubes visitantes. Por el otro, en un eje temporal, estableciéndose el antes y el después de las competiciones como los dos extremos de una herramienta que "atenaza” el espectáculo futbolístico con medidas policiales y represivas. La praxis de estos dos ejes se determina según un cálculo de peligrosidad y riesgo por parte de las autoridades estatales, cálculo que se realiza sirviéndose de una serie de variables importadas desde España en la década de 1990. Desde el punto de vista conceptual, subsiste un paradigma que guía la acción estatal y que tiende a separar la violencia de los espectadores "comunes" de la de las llamadas "barras bravas", a los barras de los "otros" barras y a las fuerzas de seguridad de todo el resto. La supuesta coherencia de esta "mini estructura social de rivalidades" registra una serie de paradojas que se han vuelto visibles para todos los actores del espectáculo menos para el Estado. Por ejemplo, si es cierto que, por un lado, el hincha “común” es sometido a un dispositivo policial riguroso de seguridad (cacheos, embudos, separación por sectores, etc), los sectores más radicalizados de las hinchadas en tanto colectivos (barras) negocian sistemáticamente, antes y después de cada espectáculo, una “adaptación” de los límites del dispositivo de seguridad con las autoridades de clubes, policiales y estatales. ${ }^{10}$

Para los actores directamente vinculados con su organización, los operativos de seguridad fracasan como consecuencia de una sociedad que estaría dominada por "malos policías", "dirigentes corruptos", "hinchas violentos e irracionales" y una legislación que "no es respetada”.

9 Eduardo Archetti (2005) ha mostrado que dicha disputa se ha zanjado en nuestro país precisamente en los cantos y en el llamado folklore o fiesta de la tribuna -lo carnavalesco- hasta la década de los 60', para pasar a partir de allí cada vez más a resolverse por la vía del ejercicio de formas de violencia material y simbólica. La violencia se ha trasformado desde entonces, como bien ha mostrado Alabarces (2014), en una cuestión que entraña a "la cultura popular y el gesto que la suprime".

10 Para los hinchas que forman parte de agrupamientos colectivos la policía no aplica con el mismo rigor los controles, entienden que las barras establecen ciertos diálogos, acuerdos y/o atajos en el ingreso al estadio que el espectador común no posee. 
Con este diagnóstico, el modelo de seguridad aplicado en las últimas décadas en el fútbol ha contribuido a naturalizar y construir el mismo como una experiencia donde la violencia -material y simbólica- se encuentra aceptada. Actualmente en el fútbol hay un clima de violencia. El hincha de fútbol es construido, en parte por el operativo, como un sujeto violento, rótulo del cual no puede distanciarse y difícilmente pueda dejar de reproducir en sus prácticas una vez que se lo significa como tal. De alguna forma, la llamada "cultura del aguante" (ALABARCES, 2004) se ha extendido como modelo hegemónico de un "comportamiento esperado" de los espectadores en los estadios de fútbol (GODIO \& ULIANA, 2015). Otro ejemplo de ello es el de los cánticos estigmatizantes y altamente despectivos del "otro" que aún hoy, sin publico visitante, se escuchan en los estadios de fútbol. Las barras se transforman en "usinas" privilegiadas en la producción de mensajes culturales que sostienen la violencia como eje de su vínculo con la otredad, con un enemigo al que hay que silenciar, excluir e inclusive "matar". Ese y otro tipo de "mensajes" más explícitamente violentos dominados por las barras -una vez que le son retirados derechos y obligaciones al resto de los espectadores- se vuelven hegemónicos o por lo menos dominantes.

Es así que las medidas de control que se implementan en los estadios están elaboradas y pensadas específicamente para contener a los hinchas potencialmente más violentos, sin distinguir entre al menos cuatro grandes categorías de aficionados en los estadios argentinos: plateístas, hinchas "activos" e "militantes", y miembros de la denominada "barra brava" (SEGURA TREJO \& MURZI, 2015). Esta orientación de los operativos de seguridad convierte a todo asistente al estadio en un criminal en potencia y lo despoja de su condición de espectador/actor partícipe de un hecho público/ritual como el fútbol. De este modo, paradójicamente, mientras que las violencias en sus formas materiales y simbólicas se refieren a hechos y actores plurales, el diseño del operativo de seguridad piensa al sujeto hincha de manera homogénea y simplificadora.

¿Cómo avanzar en la búsqueda de reemplazar los sentidos de la violencia por otros que no la impliquen? Es decir, ¿cómo producir otras expectativas en los asistentes a los estadios de fútbol que no sea aquello que Sodo (2012) llamó ambiente de violencia?

\section{HACiA UNA NUEVA EXPERIENCIA DE LA SEGURIDAd EN LOS ESTADIOS DE FÚTBOL}

En la provincia argentina de Santa Fe existen dos tipos de prácticas asociadas a la violencia que han crecido exponencialmente durante los últimos años. Por un lado, el delito complejo y el crimen organizado protagonizado por la barra que ha provocado numerosas muertes en enfrentamientos por el control territorial de negocios asociados a los partidos de fútbol, pero también a la venta de drogas. Por el otro, las acciones de violencia de los hinchas (no necesariamente barras), por ejemplo arrojar objetos al árbitro ante el descontento por un fallo o increpar y/o agredir a los jugadores o dirigentes del equipo rival que presencian el encuentro, entre muchas otras. Esto último ha provocado la suspensión o imposibilidad de organizar espectáculos futbolísticos en muchas ocasiones.

Es en este contex to que la Dirección de Coordinación de Seguridad en Competencias Deportivas y Espectáculos Masivos de la Provincia ha comenzado a plantearse la necesidad de generar condiciones para la construcción de un nuevo paradigma de seguridad. Durante los primeros pasos dados en esta dirección se apunta a cambiar la forma en que se piensa el problema, intentando abandonar una mirada que observa como causal exclusivo de la violencia en los estadios a la "irracionalidad" 
de las rivalidades entre clubes y equipos ${ }^{11}$. La nueva perspectiva, que posee un fuerte basamento científico, y el uso de resultados de investigaciones realizadas desde casi dos décadas a esta parte por las universidades públicas y agencias de investigaciones, propone incorporar el concepto de cultura -en un sentido antropológico de significados sociales, relaciones entre actores y prácticas sociales- como variable de autocontrol del comportamiento de los hinchas en los estadios para, conjuntamente, disminuir el nivel de militarización y saturación policial que se da en torno al espectáculo futbolístico.

Esta nueva visión sobre el problema propone abandonar la mirada exclusivamente policial sobre el asunto, que prioriza el control y eventualmente la sanción de las acciones violentas de los hinchas. Se propone pasar a una situación en donde el Estado, a través de las herramientas de las políticas públicas, sea el promotor de prácticas y discursos que asociados a la sociabilidad, la solidaridad y la tolerancia como ejes discursivos, recreen la pasión y la potente capacidad de autorrepresentación que los hinchas despliegan en los estadios. Disminuir la hegemonía de los lenguajes, discursos y prácticas asociadas a la violencia en los estadios es posible para el Estado, concentrándose en el fortalecimiento y la circulación de otros significados y simbologías que no estén asociados a la violencia.

Entre las acciones que se encuentran en proceso de implementación se encuentra la de priorizar el trabajo con uno de los actores centrales del fútbol y las instituciones que lo contienen: los agrupamientos de hinchas (llamados según el club subcomisiones de hinchas, peñas, filiales o agrupaciones). Los agrupamientos de hinchas son muy diversos y poseen distintos grados de institucionalización, pero realizan diferentes acciones como, por ejemplo, darle color a la tribuna los días de partido, funcionar como un espacio de sociabilidad para los que participan, ayudar al mantenimiento y ampliación de las instalaciones del club con aportes económicos, difundir los valores y la historia del club, participar de la vida política -mayoritariamente por fuera de la contienda electoral-, "producir" nuevos simpatizantes, realizar trabajos de contención social en barrios populares, entre otras. Bajo este tipo de modalidades de construcción colectiva los clubes cimentan sus identidades y buscan producir culturalmente sus particularidades.

La tarea del Estado deberá ser la de estimular la creación de nuevos significados rituales, buscando recuperar y amplificar muchos de los ya existentes. El objetivo deberá ser poner en disputa nuevos escenarios discursivos a los actuales sentidos hegemónicos de las "barras bravas". Se trata de crear un nuevo "campo de posibilidades" (VELHO, 1987) para los actores sociales que intervienen en los espectáculos y en la organización institucional de los clubes con eje en un paradigma de mayor participación y visibilidad a los agrupamientos de hinchas con distinto grado de institucionalidad. Pero también disminuir la densidad y cantidad del operativo policial en los estadios. Es posible tomar como referencia acciones puestas en marcha en el fútbol uruguayo, especialmente en lo que se refiere al accionar estatal en relación a la concepción de los comportamientos y acciones de los hinchas en los estadios, afirmando un mayor grado de responsabilidad en términos de derechos y obligaciones de todos los actores -incluidas las fuerzas de seguridad- en su condición de ciudadanos.

11 Para el caso de los cuatro clubes "grandes" de la provincia, se observa que en Rosario Central y en Colón la presencia de sus "rivales" clásicos se encuentra algo más disipada, la identidad se construye en torno a una idea de grandeza; por ejemplo, los hinchas canallas identifican al equipo con la ciudad de Rosario: "Somos la ciudad", dice la leyenda. En el caso de los hinchas sabaleros, el relato se construye en torno a un mundo popular y masivo, presente por ejemplo en la frase "Colón es pueblo". En los equipos de Newell's y Unión las marcas respecto del "otro" (Central y Colón) aparecen más explícitas y entonces la rivalidad se manifiesta más marcadamente, tomando una presencia constante y alcanzando en los relatos una mayor agresividad. 
Se ha consensuado en los siguientes cinco ejes de interpretación/acción:

1. El foco de una política pública que procure una disminución de la violencia deberá consistir en dar visibilidad y potenciar el actual trabajo de los agrupamientos de hinchas y otros colectivos sociales vinculados al fútbol. El camino propuesto se orienta a trabajar con estos actores en la búsqueda por introducir nuevos sentidos en las canchas, lenguajes, prácticas y retóricas que recreen una visión crítica de la "cultura del aguante", que hoy resulta hegemónica en las tribunas y que encuentra un actor privilegiado de su producción y circulación en las "barras”.

2. La rivalidad es un hecho constitutivo de las identidades de los equipos y clubes de la Provincia. Por lo tanto, el diseño de medidas de seguridad que se impulse desde el Estado debe tener claro y presente el respeto por las lógicas de identidad de la alteridad. Contrariamente, las actuales formas de control de la violencia operan sobre una concepción general que entiende que la rivalidad es causal excluyente de la violencia; el retiro del visitante de los estadios significa la exacerbación paroxística y extrema de esta concepción del problema.

3. Parte de las dificultades con los operativos de seguridad residen en la trasgresión de los hinchas hacia las normas y controles y en el despojo de la condición de ciudadanos que imponen los operativos. Trabajar en el fortalecimiento de la ciudadanía de los hinchas en tanto sujetos portadores de derechos y obligaciones puede ser una clave para componer la relación y responsabilizar a los hinchas de sus comportamientos. En relación a la línea investigativa, se deberán profundizar las indagaciones sobre las percepciones de los hinchas respecto de la policía y del operativo de seguridad.

4. Los hinchas de fútbol son "expertos" en acto en materia de seguridad debido a su condición de actores centrales del espectáculo futbolístico. Habilitar instancias institucionales de diálogo, consultas y extracción de sus saberes puede ser un aporte muy significativo a la construcción de la seguridad en el fútbol. En términos de investigación, deberá ser una dimensión a explorar los saberes y experiencias de los hinchas.

5. La barra impone ciertas formas materiales y simbólicas de la violencia que resultan hegemónicas en el evento futbolístico. Sin embargo, es posible disminuir el ambiente de violencia imperante en el fútbol si se fomenta e institucionaliza desde las políticas públicas la participación de agrupaciones, peñas y filiales, que en tanto agrupamientos despliegan otras significaciones asociadas a la solidaridad y la participación, alejadas de la violencia. Acercar a grupos pertenecientes a distintos clubes es posible si se explota lo que todos poseen en común: la relación con el "trabajo" por el fortalecimiento del club.

La trama de la violencia que funciona como ambiente de violencia en los estadios mantiene unidos a los heterogéneos actores del fútbol de hoy; hinchas comunes, barras, hinchas pertenecientes a agrupaciones, dirigentes de clubes, plateístas, policía, funcionarios estatales y periodistas actúan y conciben la violencia en el fútbol como una situación normal y naturalizada. La actual forma del operativo de seguridad basada en la exacerbación del control policial construye y reproduce la violencia en los estadios, y además es funcional a las barras que en parte manejan un mismo lenguaje.

En este sentido, la línea de trabajo actual en la Provincia de Santa Fe intenta pensar el problema de seguridad y la violencia en el fútbol como problema de "seguridad y desarrollo social”, donde el evento espectacular, lejos de transformarse en un lugar higiénico y despojado de conflictividad, pueda producir un escenario capaz de teatralizar constructivamente los sentidos y significados que emergen en el mismo. La construcción de una política pública bajo el formato de 
programa requiere financiamiento específico, conocimiento experto y articulación entre distintas áreas estales: seguridad, cultura y desarrollo social, fundamentalmente. La participación de los hinchas organizados en el diseño es una de las claves para el éxito. La búsqueda del empoderamiento de los hinchas por la vía de la ciudadanía, los derechos y las responsabilidades que poseen, es un camino necesario para la disminución de las prácticas violentas. Apuntar únicamente a reemplazar hinchas por consumidores equivale a vaciar de contenido cultural al fútbol, en tanto espacio de producción de identidades y expresión de tramas y conflictos sociales.

Sobre la lógica de la diferenciación se despliega la singularidad, en la que de algún modo el otro siempre debe de estar presente en "mi” existencia o experiencia cotidiana de vida. Obviamente, esto supone no negar que la construcción de identidades futboleras se exprese en términos de alteridades, pues requiere explicitar que siempre hay un "otro" frente al cual diferenciarse para poder definirse como sujeto, y en cuya definición se establecen estrategias de comprender y asir el mundo en que vivimos como sujetos. Lejos de ser ficciones "creadas" por el fútbol, las identidades y los actores sociales son emergentes puestos en escena por este último. Pero los operativos han tendido obcecadamente a subsumir estas identidades en la presuposición de ser la fuente de la violencia y la intolerancia. Desmontar esta forma simplista de significar las prácticas en que las violencias se anclan y cosifican es una de las tareas posibles de las políticas públicas de seguridad en la Argentina.

\section{Bibliografía}

ALABARCES, Pablo. Héroes, machos y patriotas. El fútbol entre la violencia y los medios. Buenos Aires: AGUILAR, 2014.

Hinchadas. Buenos Aires: Prometeo, 2005.

Crónicas del aguante. Fútbol, violencia y política. Buenos Aires: Capital intelectual, 2004.

ARCHETTI, Eduardo. "Fútbol, tango y romanticismo. Entrevista con Eduardo Archetti. In: Etnográfica. Lisboa, vol. IX, Nro 2, pps. 339-346, 2005.

Masculinidades. Fútbol, polo y tango en Argentina. Buenos Aires: Antropofagia, 2003.

AUGÉ, Marc. Los «no lugares » espacios del anonimato. Barcelona: Gedisa xxx

BROMBERGER, Christian. 2004. Football: la bagatelle la plus sérieuse du monde. Paris: Pocket, 2000.

Significado de la pasión popular por los clubes de Fútbol. Buenos Aires: Universidad de Buenos Aires, 2001

Passions ordinairs. Paris: Maison dês sciences de l’homme, 1998.

Match de Football: Ethnologie d'une Passion Partisane à Marseille, Naples et Turin. Paris: Maison dês sciences de l’homme, 1995.

DEBORD, Guy. La sociedad del espectáculo. Valencia: PRE-TEXTOS, 2005.

DUNNING, Eric. El fenómeno deportivo. Estudios sociológicos sobre deporte, violencia y civilización. Buenos Aires: Paidós, 2003.

A sociedade dos indivíduos. Rio de Janeiro: Zahar, 1994.

El proceso de la civilización. Investigaciones sociogenéticas y psicogenéticas. México: FCE, 1987 
82 - RBTS - v.3, n.1, 2016

ELIAS, Norbert \& DUNNING, Eric. Deporte y ocio en el proceso de la civilización. México: FCE, 1992.

GALVAGNI, “La hinchada de uniforme”. In: Hinchadas. Buenos Aires: Prometeo, 2005.

GARRIGA, José. “Soy macho porque me la aguanto”. In: Hinchadas. Buenos Aires: Prometeo, 2005.

GIL, Gastón. “La pasión según Aldosivi: el “otro” y los combates por la identidad”. In: Horiz. Antropológicos 14 (30): 137-164, 2008.

Fútbol e identidades locales. Buenos Aires: Miño y Dávila, 2002.

GODIO, Matías \& ULIANA, Santiago. "Separar, dividir y mortificar. Los dispositivos culturales de seguridad en los estadios de fútbol argentino”. In: GARRIGA ZUCAL, José. Violencia en el fútbol. Investigaciones sociales y fracasos políticos (org.). Buenos Aires: GODOT, 2015.

GODIO, Matías. "Somos hombres de platea. A sociedade dos dirigentes e as formas elementares do poder e da politica no futebol argentino". Tesis de Doctorado. Florianópolis: UFSC, 2010. Disponible en: https:// repositorio.ufsc.br/handle/123456789/93989

GOFFMAN, Erving. Internados. Ensayos sobre la situación social de los enfermos mentales. Buenos Aires: Amorrortu Editores, 1992.

GUTTMANN, Allen. From Ritual to Record. The Nature of Modern Sports. New York: Columbia University Press, Nueva York, 2004

KUMAR, Raúl. "Das bancadas aos sofás da Europa”. In: NEVES, José \& DOMINGOS, Nuno (org.). $A$ época do futebol. Lisboa: Zahirió \& Alvim, 2004.

MIGNON, P. La Passion du Football. Paris: Odile Jacob, 1998.

SEGURA TREJO, Fernando \& MURZI, Diego. Alternativas europeas comparadas de gestión de la seguridad y la violencia en los estadios de fútbol: tres enfoques y aplicaciones diferentes. ¿Qué se puede aprender? In: GARRIGA ZUCAL, José. Violencia en el fútbol. Investigaciones sociales y fracasos políticos (org.). Buenos Aires: xxx

RIAL, Carmen. "Porque todos os 'rebeldes' falam português? A circulação de jogadores brasileiros/sulamericanos no exterior, ontem e hoje”. In: CARMO, Renato Miguel do \& SIMÕES, José Alberto (orgs.). A produção das Mobilidades - Redes, Espacialidades e Trajectos. Lisboa: Imprensa de Ciências Sociais - ICS. pp. 203-224, 2009.

"Rodar: a circulação dos jogadores de futebol brasileiros no exterior". In: Horizontes antropológicos [online],14 (30), 2008.

ULIANA, Santiago; SUSTAS, Sebastián; MURZI, Diego. Análisis de los factores de muerte y violencia en el fútbol argentino. In: GODIO, Matías \& ULIANA, Santiago (orgs). Fútbol y Sociedad. Buenos Aires: EDUNTREF, 2011.

VELHO, Gilberto. Individualismo e cultura. Rio de Janeiro, ZAHAR, 1987. 